\title{
Report on revision of the Chinese 1992 staging system for nasopharyngeal carcinoma
}

\author{
Chinese Committee for Staging of Nasopharyngeal Carcinoma
}

Received: 25 November 2012 / Accepted: 2 January 2013 /Published online: 2 February 2013

(C) Springer-Verlag Berlin Heidelberg 2013

\begin{abstract}
With the advances of diagnostic imaging and radiation therapy technology, the limitations of the Chinese 1992 staging system for nasopharyngeal carcinoma (NPC) become obvious, and the revision of this system was clearly needed. On December 16, 2008, the Chinese Committee for Staging of Nasopharyngeal Carcinoma (CCSNPC) was inaugurated in Guangzhou, China, with the purpose of establishing a platform for the study of the Chinese staging system and ensuring the continuity of the work of NPC staging research. Data from published studies on staging of NPC were collected and reviewed. After extensive evaluation and discussion, the Chinese 2008 staging system for NPC, which was a consensus based on evidence-based medicine and revisions made on the Chinese 1992 staging system, was recommended by CCSNPC. Changes of the staging system including emphasizing the status of MRI in diagnosis and staging; classification of parapharyngeal involvement, cranial nerve involvement, and retropharyngeal lymph node involvement; and T classification in the new system were simplified as well. CCSNPC also proposed a new criterion of the $\mathrm{N}$ category. This review discusses the rationale and bases of our primary revisions of this system and proposes an updated system, named the Chinese 2008 staging system for NPC. Further investigations are needed to confirm the effectiveness as well as to provide basis for further improvement of this system.
\end{abstract}

Keywords Nasopharyngeal carcinoma $\cdot$ The Chinese 1992 staging system · The Chinese 2008 staging system . $\mathrm{T}$ classification $\cdot \mathrm{N}$ classification

\section{J. Pan $(\bowtie)$}

Department of Radiation Oncology, Cancer Hospital of Fujian

Medical University, No. 420, Fuma Road, Jinan District,

Fuzhou 350014, China

e-mail: panjianji@126.com

\section{Introduction}

Cancer staging plays one of the most important roles in the management of cancer. It forms the basis for understanding the changes in cancer incidence in a population, extent of disease at initial presentation, and the overall impact of improvements in cancer treatment. Staging also forms the base for defining groups for inclusion in clinical trials. More importantly, staging provides patients and their physicians with the critical benchmark for defining prognosis, the likelihood of disease control, and the basis for determining the best treatment approach for their diseases.

Nasopharyngeal carcinoma (NPC) is a type of malignancy with extremely imbalanced endemic distribution. It is diagnosed in many countries and regions; however, China, especially its southern part, has the highest incidence. In addition, the majority of NPC patients diagnosed in Southern China are nonkeratinizing undifferentiated carcinoma, while keratinizing squamous cell carcinoma is the predominant subtype in nonendemic regions [1]. Due to the prevalence of the disease and the large number of patients studied, Chinese experts designed a staging system based on the data of China in 1992, namely the Chinese 1992 staging system (i.e., the Fuzhou staging system). It was adopted and recommended by the Chinese Association of Radiation Oncology and other national professional associations and has been widely used in mainland China since 1992 [2]. Early studies have successfully validated the staging system $[3,4]$. However, with the advances of diagnostic imaging and radiation therapy technology, as well as treatment strategy, such as the utilizing of 3dimensional conformal radiotherapy (3D-CRT), intensitymodulated radiotherapy (IMRT), chemotherapy, and MRI, the limitations of the Chinese 1992 staging system become obvious. Revision of the Chinese 1992 staging system was clearly needed. On December 16, 2008, the Chinese Committee for Staging of Nasopharyngeal Carcinoma (CCSNPC) 
(Appendix) was inaugurated in Guangzhou, China, aimed to build a platform for the study of the Chinese staging system and ensure the continuity of the work of NPC staging research. After extensive discussion, the Chinese 2008 staging system for NPC, which was a consensus based on evidence-based medicine and primary revisions made on the Chinese 1992 staging system, was recommended by this committee.

In this report, we discuss the rationale and bases of our revision of the 1992 Chinese Nasopharyngeal Carcinoma Staging System. A detailed discussion on the recent evolution in the diagnosis and treatment of NPC that may have substantial influence on prognoses as well as their effects on staging is provided. And, the 2008 edition of the Chinese NPC Staging System is proposed.

\section{The Chinese 1992 staging system for NPC and its limitations}

\section{Background}

The Chinese 1992 staging system (Table 1) was developed based on the retrospective data of Sun Yat-sen University reported by Min et al. [2]. Four hundred and twenty-one cases of NPC treated at their institution during 1985 to 1987 with computed tomography (CT) scan before initial radiotherapy were analyzed. Important prognostic factors that formed the basis of the staging system were screened out by means of Cox model and clinical experiences. This staging system was valuable in predicting patients' prognoses and improving target localization for radiation therapy since its publication. However, there are evident limitations in the modern era of nasopharyngeal cancer care, with more advanced technology of diagnosis (e.g., MRI) and treatment (e.g., chemoradiation for locally advanced disease, IMRT, or 3D-CRT).

\section{Limitations}

\section{Based only on CT images}

The advent of CT in the mid-1970 s led to substantially better staging than conventional polytomography and supported more conformal treatment planning. MRI, which was introduced in the mid-1980 s, has the advantages of better tissue contrast, multiplanar capacity, and lack of radiation and bone beam-hardening artifacts, when compared to CT. Studies have shown that MRI allows for a more accurate evaluation of the primary tumor extent, especially for the early disease, and lymphadenopathy than does CT [5-8], and it shifts the classification in either direction in more than $30 \%$ of patients, leading to changes in treatment strategies
Table 1 The Chinese 1992 staging system

\begin{tabular}{|c|c|}
\hline \multicolumn{2}{|l|}{$\mathrm{T}$ - primary tumor } \\
\hline $\mathrm{T} 1$ & Tumor confined to the nasopharynx \\
\hline $\mathrm{T} 2$ & $\begin{array}{l}\text { Involvement of nasal cavity, oropharynx, soft } \\
\text { palatine, anterior cervical vertebrae soft tissue, } \\
\text { and parapharyngeal space extension before SO } \\
\text { line }^{\mathrm{a}}\end{array}$ \\
\hline $\mathrm{T} 3$ & $\begin{array}{l}\text { Extension over SO line, involvement of anterior } \\
\text { or posterior cranial nerves alone, skull base, } \\
\text { pterygoprocess zone, and pterygopalatine fossa }\end{array}$ \\
\hline $\mathrm{T} 4$ & $\begin{array}{l}\text { Involvement of both anterior and posterior } \\
\text { cranial nerves, paranasal sinus, cavernous } \\
\text { sinus, orbit, infratemoral fossa, and direct } \\
\text { invasion of first or second cervical vertebra }\end{array}$ \\
\hline \multicolumn{2}{|c|}{$\mathrm{N}$-regional lymph nodes } \\
\hline N0 & No enlarged lymph node \\
\hline N1 & $\begin{array}{l}\text { The diameter of upper neck lymph node }<4 \mathrm{~cm} \text {, } \\
\text { movable }\end{array}$ \\
\hline $\mathrm{N} 2$ & $\begin{array}{l}\text { Lower neck lymph node or the diameter between } \\
4 \text { and } 7 \mathrm{~cm}\end{array}$ \\
\hline N3 & $\begin{array}{l}\text { Supraclavicular lymph node or the diameter } \\
>7 \mathrm{~cm} \text { or fixed or skin infiltration }\end{array}$ \\
\hline \multicolumn{2}{|c|}{$\mathrm{M}$-distant metastasis } \\
\hline M0 & Absence of distant metastasis \\
\hline M1 & Presence distant metastasis \\
\hline \multicolumn{2}{|l|}{ Stage grouping } \\
\hline \multicolumn{2}{|l|}{ Stage I T1 N0 M0 } \\
\hline $\begin{array}{l}\text { Stage II T2 N0- } \\
1 \mathrm{M} 0\end{array}$ & T1-2 N1 M0 \\
\hline $\begin{array}{l}\text { Stage III T3 N0- } \\
2 \mathrm{M} 0\end{array}$ & T1-3 N2M0 \\
\hline $\begin{array}{l}\text { Stage IVA T4 any } \\
\quad \mathrm{N}, \mathrm{M} 0\end{array}$ & Any T N3, M0 \\
\hline $\begin{array}{l}\text { Stage IVB any } \mathrm{T}, \\
\text { any } \mathrm{N}, \mathrm{M} 1\end{array}$ & \\
\hline
\end{tabular}

${ }^{a}$ SO line, The line connecting the styloid process to the midpoint on the posterior edge of the great occipital foramen. The border between upper and lower neck is the lower margin of the cricoid cartilage

for NPC [9]. As such, staging according to the evidences of MRI images becomes standard.

\section{No clear definition of anatomical structure and consistent criteria of invasion}

Some anatomical structures were not fully addressed in the Chinese 1992 staging system. For example, the definition of invasion beyond the nasal cavity and the classification of retropharyngeal lymph nodes were not mentioned. In addition, criteria of nasal cavity and infratemporal fossa invasion varied in different institutions. Moreover, descriptions of parapharyngeal space involvement and lymph node metastases were subjective and may be partly based on the physicians' experience and personal judgment. 


\section{Change of treatment strategies and technologies}

The implementation of 3D-CRT, particularly IMRT, has allowed high-dose delivery to tumors while sparing normal tissues, and the utilization of MRI is expected to be complementary in delineating the gross tumor volume contours and ensuring correct coverage of the primary tumor and nodal metastasis [10]. The combination of chemoradiotherapy further improves the outcome of NPC, especially in locoregionally advanced diseases [11]. The 5-year overall survival rate approaches $80 \%$ in recent reports, which has been significantly improved as compared to $50 \%$ in the early $1990 \mathrm{~s}$; prognostic factors for NPC have significantly changed as well [10-12].

In summary, the Chinese 1992 staging system may not predict the outcome of NPC well after definite treatment in the new era and has become obsolete. In addition, due to the high incidence and the different pathological types of NPC between China and nonendemic regions, we considered that the UICC staging system, which is widely used in other countries, may have lack of flexibility for Chinese patients. After adequate discussion, Chinese experts recommended a new staging system by revising the previous system in 2008 .

\section{The development of the Chinese 2008 staging system}

On December 16, 2008, CCSNPC was inaugurated in Guangzhou, China, with the purpose of building a platform for the study of the Chinese staging system and ensuring the continuity of the work of NPC staging research. The committee made a preliminary revision of the Chinese 1992 staging system and concluded the first draft of the Chinese 2008 staging system (Table 2). Changes of the staging system were listed in Table 3 and detailed as follows.

MRI was considered as the preferred imaging modality for NPC diagnosis and staging

Due to the particular anatomic position of the nasopharynx, primary tumor extension can be only evaluated by CT or MRI. When compared to CT, MRI has the advantages of better tissue contrast, multiplanar capacity, and lack of radiation and bone beam-hardening artifacts. It can clearly identify between a tumor that extends to the sinus and obstructive inflammation. In addition, it could well distinguish retropharyngeal lymph node metastasis from primary tumor extension to the parapharyngeal space. Moreover, its sensitivity in detecting tumor involvement in the parapharyngeal space, oropharyngeal space, retropharyngeal lymph node, bony skull base, cavernous sinus, sinuses, cervical vertebra, and temporal fossa, and tumor extension across the nasal cavity have been reported to be significantly higher than those of CT [5-8]. PET/CT has an advantage of
Table 2 The Chinese 2008 staging system

\begin{tabular}{|c|c|c|}
\hline \multicolumn{3}{|c|}{$\mathrm{T}$ - primary tumor } \\
\hline $\mathrm{T} 1$ & \multicolumn{2}{|c|}{ Tumor confined to the nasopharynx } \\
\hline $\mathrm{T} 2$ & \multicolumn{2}{|c|}{ Nasal cavity, oropharynx, parapharyngeal extension } \\
\hline $\mathrm{T} 3$ & \multicolumn{2}{|c|}{ Skull base, medial pterygoid muscle extension } \\
\hline $\mathrm{T} 4$ & \multicolumn{2}{|c|}{$\begin{array}{l}\text { Cranial nerves, paranasal sinuses, masticator space } \\
\text { excluding medial pterygoid muscle, intracranial } \\
\text { (cavernous sinus, dural meninges) extension }\end{array}$} \\
\hline \multicolumn{3}{|c|}{$\mathrm{N}$-regional lymph nodes } \\
\hline No & \multicolumn{2}{|c|}{ No regional lymph node metastasis } \\
\hline N1a & \multicolumn{2}{|c|}{ Retropharyngeal lymph node involvement } \\
\hline $\mathrm{N} 1 \mathrm{~b}$ & \multicolumn{2}{|c|}{$\begin{array}{l}\text { Unilateral level Ib, II, III, and Va involvement, } \\
\text { and the maximum diameter } \leq 3 \mathrm{~cm}\end{array}$} \\
\hline $\mathrm{N} 2$ & \multicolumn{2}{|c|}{$\begin{array}{l}\text { Bilateral level Ib, II, III, and Va involvement, or the } \\
\text { maximum diameter }>3 \mathrm{~cm} \text { or with extranodal } \\
\text { neoplastic spread }\end{array}$} \\
\hline N3 & \multicolumn{2}{|c|}{ Level IV, $\mathrm{Vb}$ involvement } \\
\hline \multicolumn{3}{|c|}{$\mathrm{M}$ - distant metastasis } \\
\hline M0 & \multicolumn{2}{|c|}{ Absence of distant metastasis } \\
\hline M1 & \multicolumn{2}{|c|}{ Presence distant metastasis } \\
\hline \multicolumn{3}{|c|}{ Stage grouping } \\
\hline Stage I & \multicolumn{2}{|l|}{ T1 N0 M0 } \\
\hline Stage II & T1 N1a-1b M0 & T2 N0-1b M0 \\
\hline Stage III & T1-2 N2 M0 & T3 N0-2 M0 \\
\hline Stage IVA & $\mathrm{T} 1-3 \mathrm{~N} 3, \mathrm{M} 0$ & T4 N0-3, M0 \\
\hline Stage IVB & Any T, any N, M & \\
\hline
\end{tabular}

detecting local, regional, and distant metastases in our body. However, disadvantages such as a lower resolution and inferior accuracy for $\mathrm{T}$ and $\mathrm{N}$ classification as compared to MRI must be considered [13, 14]. Thus, MRI was considered as the most favorable imaging modality for the Chinese 2008 staging system.

Table 3 Summary of changes

-MRI was considered as the preferred imaging modality for NPC diagnosis and staging.

-Patients with parapharyngeal involvement including prestyloid space and poststyloid space were classified as $\mathrm{T} 2$

-Infratemporal fossa was removed, while masticator space was added to T4.

-Any cranial nerves involvement was reclassified as T4.

-T classification was simplified; some structures, such as anterior cervical vertebrae soft tissue, soft palatine, pterygopalatine fossa, orbit, and cervical vertebrae, were excluded.

-Patients with retropharyngeal lymph node involvement were classified as N1a, either unilateral or bilateral.

- $\mathrm{N}$ classification was based on MRI images and judged according to the criteria of RTOG, and the traditional nomenclature would no longer be needed; in addition, the site, size, laterality, and extranodal neoplastic spread were enrolled in the criteria of $\mathrm{N}$ category. 
Patients with parapharyngeal space involvement including prestyloid space and poststyloid space were classified as T2

Parapharyngeal space involvement was described as tumor invasion across buccopharyngeal fascia. The buccopharyngeal fascia is parallel to the carotid sheath, and along its medial aspect, the prevertebral fascia gives off a thin lamina, the buccopharyngeal fascia, which closely invests the constrictor muscles of the pharynx and is continued forward from the constrictor pharyngis superior onto the buccinator. It is attached to the prevertebral layer by loose connective tissue only, and thus, an easily distended space, the retropharyngeal space, is found between them.

Data from Min et al. [2] indicated that within carotid area, tumors that extend beyond the line that connects the styloid process to the midpoint on the posterior edge of the great occipital foramen (SO line) have worse prognosis than those anterior to the SO line. Thus, primary tumor extension over the SO line was defined as T3 in the Chinese 1992 staging system, while parapharyngeal space extension before the SO line was specified as T2. In the conventional radiotherapy era using the face and neck as a separate field, the posterior border would be put forward in order to avoid excessive exposure of the brainstem and the spinal cord, which may consequently cause insufficient dose in the poststyloid space. Parapharyngeal tumor involvement, especially in the carotid area, is a stronger predictor for poor prognosis $[15,16]$. Even after the introduction of faciocervical fields, the prognostic value of parapharyngeal involvement remained varied in different studies, mainly because the boost in the parapharyngeal area was not adopted in all the institutions [17-21]. However, with the prevailing use of MRI, CT simulation, and 3D-CRT or IMRT, the possibility of inadequate dose in this area was reduced, and parapharyngeal space extension per se no longer predicts disease outcome $[5,22,23]$.

In a study reported by Gao et al. [24] on the Chinese 1992 staging system based on CT images, a total of 915 patients were enrolled. The authors reclassified patients whose carotid sheath area was totally occupied by the tumor as $\mathrm{T} 2$, and the results indicated that the survival of patients with $\mathrm{T} 2$ and $\mathrm{T} 3$ diseases differed more significantly after the reclassification. In addition, Mao et al. [25] found that the risk of local recurrence of T3 disease with carotid sheath area involvement was comparable to that of T2 disease in their study based on MRI; they enrolled a total of 924 patients and concluded that patients with parapharyngeal involvement should be classified in the same T category. Based on these studies, the Chinese 2008 staging system described those with parapharyngeal involvement as $\mathrm{T} 2$ disease.
Infratemporal fossa was removed while masticator space was added to $\mathrm{T} 4$

The infratemporal fossa is an irregularly shaped cavity, situated below and medial to the zygomatic arch. Harnsberger defined the infratemporal fossa as the nasopharyngeal portion of the masticator space [26]. It contains the masticator muscles, the internal maxillary vessels, and the mandibular and auriculo-temporal nerves, and the foramen ovale and foramen spinosum open through the roof of the fossa [27]. The infratemporal fossa was used to describe the extent of lateral invasion from the primary lesion in the Chinese 1992 staging system and was classified as T4. However, infratemporal fossa is less frequently used in radiologic practice to describe nasopharyngeal disease extension.

The masticator space describes the anatomic region below the middle cranial fossa. This term is generally preferred in the radiology community and has the advantage that there are clear anatomic boundaries defined by fascial planes defining it. It is divided into two parts by the zygomatic arch, including the supratemporal fossa and infratemporal fossa. In order to avoid confusion of the concepts and study the role of the masticator space further, the masticator space was used in the Chinese 2008 staging system as a T4 descriptor instead of infratemporal fossa in the Chinese 1992 staging system. The new draft emphasized that the masticator space in the staging system refers in particular to the infratemporal fossa. Its boundaries are: anteriorly, next to the fat clearance behind the maxillary sinus; posteriorly, by the superficial layer of the deep cervical fascia that covers the medial and lateral pterygoid and articular tubercle formed by the temporal bone; medially, by the lateral pterygoid plate and internal margin of the medial pterygoid; laterally, by the masseter muscle and the mandibular ramus, superiorly, under the level of the zygomatic arch. Considering the biological behavior of NPC, patients with medial pterygoid muscle extension will be classified as T3, while those with masticator space involvement excluding the medial pterygoid muscle will be grouped into T4.

Any cranial nerve involvement was reclassified as T4

The incidence of cranial nerve palsy is not unusual in patients with untreated NPC, the frequency of diagnosed cranial nerve palsy in NPC ranging from 8.0-12.4\% [28-30]. According to the Chinese 1992 staging system, involvement of anterior group cranial nerve (I, II, III, IV, $\mathrm{V}$, and VI) or posterior cranial nerve (VII, VIII, IX, X, XI, and XII) invasion was defined as T3, while involvement of both would be defined as T4. In a series of 924 patients by Mao et al. [25], $85.3 \%$ of the patients with only either anterior or posterior cranial nerve involvement presented with another staging descriptor in the T4 category; the local 
recurrence rate of $\mathrm{T} 3$ with only either anterior or posterior cranial nerve involvement was comparable with that of $\mathrm{T} 4$ disease, suggesting that all patients with any cranial nerve involvement may be reclassified as T4. Gao et al. [24] also revealed that the survival curves that differed more significantly after T3 disease with cranial nerve involvement be reclassified as T4. Based on these data, in the Chinese 2008 staging system, all cases of cranial nerve involvement will be classified as $\mathrm{T} 4$.

T classification was simplified, and some structures, such as anterior cervical vertebrae soft tissue, soft palatine, pterygopalatine fossa, orbit, and cervical vertebrae, were excluded

It has been reported that all patients with involvement of any of the following anatomy structures, including anterior cervical vertebrae soft tissue, soft palatine, pterygopalatine fossa, and orbit, must be presented with other staging criteria in the same $\mathrm{T}$ category or a higher $\mathrm{T}$ level in the Chinese 1992 staging system [25]. These results suggested that excluding the anatomy structures mentioned above in the staging system may not affect the constituent ratio of $\mathrm{T}$ category. Feng et al. [31] initiated a study of 521 patients based on MRI images; $92 \%$ of patients with anterior cervical vertebrae soft tissue involvement were in the T3 or T4 category. Chung et al. [32] also found approximately $96.1 \%$ of patients with pterygopalatine fossa involvement accompanied with intracranial extension. Moreover, it has been indicated that invasive rates of the orbit, laryngopharynx, and cervical vertebra were very low [33], and involvement of the soft palatine has been seldom reported. According to these studies, we remove anterior cervical vertebrae soft tissue, soft palatine, pterygopalatine fossa, and orbit in the Chinese 2008 staging system.

Patients with retropharyngeal lymph node metastasis were classified as N1a, either unilateral or bilateral

The majority of lymph node metastases follow the sequential and orderly fashion, with a skip metastases rate of less than $5 \%$ [34-36]. Retropharyngeal lymph nodes are considered as the first echelon of nodal spread and should be placed into the N1 category. However, it was not included in the Chinese 1992 staging system, mainly because it is difficult to distinguish retropharyngeal lymph node metastasis from primary tumor extension on CT images, and was consequently described as parapharyngeal space involvement. MRI is superior to CT in the identification of retropharyngeal lymph node metastasis, not only for detecting small-sized nodes, but also in distinguishing the extension of the primary tumor in the poststyloid space and retropharyngeal lymph nodes. A study from China using MRI for diagnosis has reported abnormal retropharyngeal nodes in excess of $70 \%$ [37], compared to approximately $50 \%$ in a series from Singapore, where CT was the predominant staging modality [38]. Evidence from these two studies and that of Ma et al. [39] indicated that patients with retropharyngeal lymph nodes alone have a risk of distant metastasis that is similar to N1 disease. These studies proposed that patients with retropharyngeal lymph node metastasis should be classified as N1 and should be independent of laterality. In the Chinese 2008 staging system, patients with retropharyngeal lymph node metastasis will be classified as N1a, either unilateral or bilateral.

$\mathrm{N}$ classification was based on MRI images and judged according to the criteria of the RTOG, and the traditional nomenclature would no longer be needed; in addition, the site, size, laterality, and extranodal neoplastic spread were enrolled in the criteria of the $\mathrm{N}$ category

The N classifications of the Chinese 1992 staging system had limitations. First, the $\mathrm{N}$ category criteria mainly depend on clinical examination, which could be highly unreliable and dependent on the experience of the physicians [40]. In addition, the definition of the supraclavicular fossa, described in the Chinese 1992 staging system, is based primarily on clinical landmarks, and there is no reliable way to define the supraclavicular fossa radiologically [41]. The introduction of new types of therapeutic interventions or new technologies may require modification of the classification and staging systems [42]. Because MRI is considered as the standard method for pretreatment diagnosis and staging [42], it is urgent to reevaluate the prognostic value of MRI-determined nodal variables for NPC.

In a retrospective study by Mao et al. [43], the MRI scans of 924 patients with histologically diagnosed nondisseminated NPC were reviewed, and distribution of the tumors was mapped using Radiation Therapy Oncology Group (RTOG) guidelines and laterality. Their results indicated that

Table 4 Criteria of measuring lymph nodes on MRI images

-With a minimum diameter of not less than $10 \mathrm{~mm}$ on the crosssectional images

- Central necrosis or rim enhancement

-More than 3 lymph nodes in a high-risk region, and at least one of the involved lymph nodes with the minimum diameter on the largest cross-sectional images not less than $8 \mathrm{~mm}$ (high-risk region was described as follows: level II for N0, the next level of that with lymph node involvement for $\mathrm{N}+$ patients

-Extranodal neoplastic spread, such as irregular enhancement at the edge of the lymph node, part or all of the fat space disappears, and lymph node convergence

-Retropharyngeal lymph nodes: with the minimum diameter on the largest cross-sectional images not less than $5 \mathrm{~mm}$ 
variables of involved regional nodes including level, laterality, and extranodal neoplastic spread were independent prognostic factors for NPC and suggested that these three variables should be included in the $\mathrm{N}$ category for staging. The proposed N classification of NPC using RTOG guidelines based on MRI was highly predictive and may provide a more objective method for staging NPC. This study also indicated that patients with level IV and supraclavicular fossa involvement had a significant increase in hazard ratios. Thus, extension to level $\mathrm{Vb}$, which was part of the supraclavicular regions, was defined as N3, whereas level Va was classified as N2 in the Chinese 2008 staging system for NPC. Partition of cervical lymph nodes is defined according to the guidelines of DAHANCA EOTRC, GORTEC, the NCIC, and RTOG [41]. This definition was based on the data published in 2003, in which CT-based delineation of lymph node levels and related CTVs in the node-negative neck was evaluated [44].

Extranodal neoplastic spread (ENS) has been reported to be one of the independent prognostic factors for NPC in the study of Mao et al. [43]; however, whether nodal size was independently significant in NPC has not been fully addressed [45-47]. Lee et al. [45] found that the maximum diameter (MAD) of a lymph node was independently significant in predicting survival, but both Heng et al. [46] and Teo et al. [47] found that the MAD of a lymph node was not a significant prognostic factor. But, in the Chinese 2008 staging system, the size of the lymph node was retained, and $3 \mathrm{~cm}$ was used as a benchmark. One potential reason for the discrepancy between the results of these studies is that the measurement of lymph node size in all these studies was based on clinical palpation [45-47]. Using MRI, MAD can be measured more accurately than the palpation-based greatest dimension, which might contain the fusion of multiple nodes. In addition, the MAD of the lymph node was significantly interrelated with ENS, as indicated in the study of Don et al. [48]. The authors found that $23 \%$ of metastatic nodes measuring $<2 \mathrm{~cm}, \sim 50 \%$ of those measuring 2 to $3 \mathrm{~cm}$, and $75 \%$ of those measuring $>3 \mathrm{~cm}$ have ENS in patients with primary squamous cell carcinoma of the head and neck. Moreover, the identification of ENS for NPC was based on imaging but not pathology, thus more subjective than identification of other features of malignancy, such as nodal size measuring on MR images, and resulted in a wider variation in interpretation. As such, patients with the lymph nodes larger than $3 \mathrm{~cm}$ are recommended to be reclassified as N2 in the Chinese 2008 staging system, using the MAD of the lymph node measured on the cross-sectional MRI images as the standard. More information on measurement of the lymph nodes is detailed in Table 4 [49].

\section{Conclusions}

The Chinese 2008 staging system for nasopharyngeal carcinoma, which is based on the more advanced imaging techniques and more recently published clinical evidences, is considered to be more reasonable than the Chinese 1992 staging system. However, further studies are needed to directly confirm the usefulness and provide basis for further improvement of the system.

Conflict of interest The authors declare that they have no conflict of interest.

\section{Appendix}

Chinese Committee for Staging of Nasopharyngeal Carcinoma: Guozhen Xu, MD (Consultant), Cancer Hospital, Chinese Academy of Medical Science; Taixiang Lu, MD (Chairperson), Cancer Center, Sun Yat-sen University; Minghuang Hong, MD (Chairperson), Cancer Center, Sun Yat-sen University; Jianji Pan, MD (Chairperson), Fujian Provincial Cancer Hospital; Jinyi Lang, MD (Vice Chairperson), Sichuang Provincial Cancer Hospital; Chaosu Hu, MD (Vice Chairperson),Cancer Hospital, Fudan University; Li Gao, MD (Vice Chairperson) Cancer Hospital, Chinese Academy of Medical Science; Rensheng Wang, MD, The First Affiliated Hospital of Fujian Medical University; Zhengchao Pi, MD, Hubei Provincial Cancer Hospital; Xiaodong Zhu, MD, Guangxi Provincial Cancer Hospital; Yiqin Zhang, MD, Jiangsu Provincial Cancer Hospital; Meng $\mathrm{Wu}, \mathrm{MD}$, Jiangxi Provincial Cancer Hospital; Feng Jin, MD, Guizhou Provincial Cancer Hospital; Xianzhao Chen, MD, Hainan Provincial Cancer Hospital; Xiaozhong Chen, MD, Zhejiang Provincial Cancer Hospital; Yunbin Chen, MD, Fujian Provincial Cancer Hospital; Xinhua Yang, MD, Shandong Provincial Cancer Hospital; Xuping $\mathrm{Xu}, \mathrm{MD}$, Hunan Provincial Cancer Hospital; Biling Liang, MD, Sun Yat-sen Memorial Hospital, Sun Yat-sen University; Jun Sui, MD, Yunnan Provincial Cancer Hospital; Shaojun Lin, MD (Secretary), Fujian Provincial Cancer Hospital; Junlin Yi, MD (Secretary), Cancer Hospital, Chinese Academy of Medical Science; Chong Zhao, MD (Secretary), Cancer Center, Sun Yat-sen University.

\section{References}

1. Vasef MA, Ferlito A, Weiss LM (1997) Nasopharyngeal carcinoma, with emphasis on its relationship to Epstein-Barr virus. Ann Otol Rhinol Laryngol 106:348-356 
2. Min HQ, Hong MH, Ma J et al (1994) A new staging system for nasopharyngeal carcinoma in China. Int $\mathrm{J}$ Radiat Oncol Biol Phys 30:1037-1042

3. Ma J, Min HQ, Hong MH et al (1999) Clinical validation of the Chinese 1992 staging system. Ai zheng 18:44-45

4. Hong MH, Mai HQ, Min HQ et al (2000) A comparison of the Chinese 1992 and Fifth-Edition International Union Against Cancer Staging Systems for staging nasopharyngeal carcinoma. Cancer 89:242-247

5. King AD, Teo P, Lam WW et al (2000) Paranasopharyngeal space involvement in nasopharyngeal cancer: detection by CT and MRI. Clin Oncol 12:397-402

6. Ng SH, Wan YL, Ko SF et al (1998) MRI of nasopharyngeal carcinoma with emphasis on relationship to radiotherapy. J Magn Reson Imaging 8:327-336

7. Chong VF, Fan YF (1996) Skull base erosion in nasopharyngeal carcinoma: detection by CT and MRI. Clin Radiol 51:625-631

8. Casselman JW (1994) The value of MRI in the diagnosis and staging of nasopharynx tumors. J Belge Radiol 77:67-71

9. Sun Y, Mao YP, Ma J et al (2007) Influences of magnetic resonance imaging on the staging system of nasopharyngeal carcinoma. Ai Zheng 26:158-163, In Chinese

10. Wang T, Riaz N, Cheng S et al (2012) Intensity-modulated radiation therapy for nasopharyngeal carcinoma: a review. J Radiat Oncol 1:129-146

11. Lee A, Ng W, Chan O et al (2012) If concurrent-adjuvant chemoradiotherapy is beneficial for locoregionally advanced nasopharyngeal carcinoma, would changing the sequence to inductionconcurrent achieve better outcome? J Radiat Oncol 1:107-115

12. Yi JL, Gao L, Huang XD (2006) Nasopharyngeal carcinoma treated by radical radiotherapy alone: ten-year experience of a single institution. Int J Radiat Oncol Biol Phys 65:161-168

13. Su Y, Zhao C, Xie CM et al (2006) Evaluation of CT, MRI and PET-CT in detecting retropharyngeal lymph node metastasis in nasopharyngeal carcinoma. Ai Zheng 25:521-525

14. Hu WH, Zhang GY, Liu LZ (2005) Comparison between PET-CT and MRI in diagnosing nodal metastasis of nasopharyngeal carcinoma. Ai Zheng 24:855-860, In Chinese

15. Wu SX, Zeng ZF, Zhao C (1998) Prognostic value of parapharyngeal space involvement in nasopharyngeal carcinoma. Ai Zheng 17:286-289

16. Sham JS, Choy D (1991) Prognostic value of paranasopharyngeal extension of nasopharyngeal carcinoma on local control and shortterm survival. Head Neck 13:298-310

17. Chua DT, Sham JS, Kwong DL (1996) Prognostic value of paranasopharyngeal extension of nasopharyngeal carcinoma: a significant factor in local control and distant metastasis. Cancer 78:202-210

18. Xiao GL, Gao L, Xu GZ (2002) Prognostic influence of parapharyngeal space involvement in nasopharyngeal carcinoma. Int J Radiat Oncol Biol Phys 52(4):957-963

19. Huang PY, Sun ZY, Xie CM (2012) Prognostic significance of the various classifications for parapharyngeal space involvement in nasopharyngeal carcinoma. Acta Otolaryngol 132:1197-207

20. Teo P, Lee WY, Yu P (1996) The prognotic significance of parapharyngeal tumor involvement in nasopharyngeal carcinoma. Radiother Oncol 39:209-221

21. Au JSK, Law DK, Foo FR et al (2003) In-depth evaluation of the AJCC/UICC 1997 staging system of nasopharyngeal carcinoma: prognostic honogeneity and proposed refinements. Int $\mathrm{J}$ Radiat Oncol Biol Phys 56:413-426

22. Kam MKM, Chau RMC, Suen J (2003) Intensity-modulated radiotherapy in nasopharyngeal carcinoma: dosimetric advantage over conventional plans and feasibility of dose escalation. Int $\mathbf{J}$ Radiat Oncol Biol Phys 56:145-157

23. Ng WT, Chan SH, Lee AW (2008) Parapharyngeal extension of nasopharyngeal carcinoma: still a significant factor in era of modern radiotherapy? Int J Radiat Oncol Biol Phys 72:1082-1089
24. Gao JM, Zeng YX, Cui NJ et al (2005) Staging 915 cases of nasopharyngeal carcinoma after simple radical radiotherapycheckout of Fuzhou staging system (1992). Ai Zheng 24:11651172, In Chinese

25. Mao YP, Hong MH et al (2007) Clinical staging of nasopharyngeal carcinoma based on MRI: suggestions for improving the Chinese '92 staging system. Ai Zheng 26:1099-1106, In Chinese

26. Harnsberger HR (1995) The masticator space. In: Harnsberger HR (ed) Handbook of head and neck imaging, 2nd ed. Mosby, St. Louis, pp 46-59

27. Mafee (2005) Nasopharyngnx. In: Mafee MF, Valvassori GE, Becker M (eds) Imaging of the head and neck, 2nd edn. Thieme, Stuttgart, pp 555-578

28. Lin L, Liang S, Li L et al (2009) Prognostic impact of magnetic resonance imaging-detected cranial nerve involvement in nasopharyngeal carcinoma. Cancer 115:1995-2003

29. Sanguineti G, Geara FB, Garden AS et al (1997) Carcinoma of the nasopharynx treated by radiotherapy alone: determinants of local and regional control. Int J Radiat Oncol Biol Phys 37:985-996

30. Cheng SH, Tsai SY, Horng CF et al (2006) A prognostic scoring system for locoregional control in nasopharyngeal carcinoma following conformal radiotherapy. Int J Radiat Oncol Biol Phys 66:992-1003

31. Feng AC, Wu MC, Tsai SY et al (2006) Prevertebral muscle involvement in nasopharyngeal carcinoma. Int J Radiat Oncol Biol Phys 65:1065-1035

32. Chung NN, Ting LL, Hsu WC et al (2004) Impact of magneticresonance imaging versus $\mathrm{CT}$ on nasopharyngeal carcinoma: primary tumor target delineation for radiotherapy. Head Neck 26:241-246

33. Han J, Shen WR, He X et al. (2006) A comparison of CT and MRI in diagnosis for nasopharyngeal carcinoma. Zhong Liu Xue Za Zhi 12:279-281

34. Sham JST, Choy MB, Wei WI et al (1990) Nasopharyngeal carcinoma: orderly neck node spread. Int J Radiat Oncol Biol Phys 19:929-933

35. King AD, Ahuja AT, Leung SF et al (1990) Neck node metastases from nasopharyngeal carcinoma: MR imaging of patterns of disease. Head Neck 2000(22):275-281

36. Sun Y, Ma J, Lu TX et al (2004) Regulation for distribution of metastatic cervical lymph nodes of 512 cases of nasopharyngeal carcinoma. Ai Zheng 23(11 Suppl):1523-1527

37. Tang L, Li L, Mao Y (2008) Retropharyngeal lymph node metastasis in nasopharyngeal carcinoma detected by magnetic resonance imaging: prognostic value and staging categories. Cancer 113:347-354

38. Tham IW, Hee SW, Yap SP (2009) Retropharyngeal nodal metastasis related to higher rate of distant metastasis in patients with $\mathrm{N} 0$ and N1 nasopharyngeal cancer. Head Neck 31:468-474

39. Ma J, Liu LZ, Tang LL et al (2007) Retropharyngeal lymph node metastasis in nasopharyngeal carcinoma: prognostic value and staging categories. Clin Cancer Res 13:1445-1452

40. Ng WT, Lee AW, Kan WK et al (2007) N-staging by magnetic resonance imaging for patients with nasopharyngeal carcinoma: pattern of nodal involvement by radiological levels. Radiother Oncol 82:70-75

41. Gregoire V, Eisbruch A, Hamoir M et al (2006) Proposal for the delineation of the nodal CTV in the node-positive and the postoperative neck. Radiother Oncol 79:15-20

42. Cooper J, Fleming ID, Henson DE (eds) (2002) American Joint Committee on Cancer manual for staging of cancer, 6th edn. JBLippincott, Philadelphia

43. Mao YP, Liang SB, Liu LZ et al (2008) The N staging system in nasopharyngeal carcinoma with Radiation Therapy Oncology Group guidelines for lymph node levels based on magnetic resonance imaging. Clin Cancer Res 14:7903-7947 
44. Gregoire V, Levendag P, Ang KK et al (2003) CT-based delineation of lymph node levels and related CTVs in the node-negative neck: DAHANCA, EORTC, GORTEC, NCIC, RTOG consensus guidelines. Radiother Oncol 69:227-236

45. Lee AW, Foo W, Law SC et al (1999) Staging of nasopharyngeal carcinoma: from Ho's to the new UICC system. Int J Cancer 84:179-187

46. Heng DM, Wee J, Fong KW et al (1999) Prognostic factors in 677 patients in Singapore with nondisseminated nasopharyngeal carcinoma. Cancer 86:1912-1920
47. Teo P, Shiu W, Leung SF et al (1992) Prognostic factors in nasopharyngeal carcinoma investigated by computertomography: an analysis of 659 patients. RadiotherOncol 23:7993

48. Don DM, Anzai Y, Lufkin RB (1995) Evaluation of cervical lymph node metastases in squamous cell carcinoma of the head and neck. Laryngoscope 105:669-74

49. Ven den Brekel MW, Stel HV, Castelijns JA et al (1990) Cervical lymph node metastasis: assessment of radiologic criteria. Radiology 177:379-384 